\title{
The Increased Burden of SARS-CoV-2 Infection in Orthopaedic Trauma Patients: Comparison of Demographics of Both the Waves of the Pandemic - An Indian Tertiary Center Experience
}

\author{
Sandeep Patel ${ }^{1}$. Shahnawaz Khan ${ }^{1}$. Sameer Aggarwal ${ }^{1}$. Vishal Kumar ${ }^{1}$. Siddhartha Sharma ${ }^{1}$. \\ Mandeep Singh Dhillon ${ }^{1}$
}

Received: 30 June 2021 / Accepted: 2 September 2021 / Published online: 13 September 2021

(C) Indian Orthopaedics Association 2021

\begin{abstract}
Background The second wave of SARS-CoV-2 pandemic has posed new challenges in the management of Orthopedic trauma patients due to the overburdened healthcare facilities and we aim to present the differences in demographics between the first wave and the initial part of the second wave.

Methodology This study was a retrospective cross-sectional study of our trauma registry from June 19, 2020 to October 13, 2020 (1st study period) and from April 1, 2021 to the first week of May 2021 (2nd study period). We looked into differences in the following three parameters: (1) infection rate among Orthopaedic patients in the first and second study period of SARS-CoV-2 pandemic, (2) infection rate among the Health Care Workers and (3) hospital-acquired SARS-CoV-2 infections in admitted Orthopaedic trauma patients.

Results 35 out of 852 patients (4.1\%) were positive for SARS-CoV-2 infection in 1st study period as compared to 48 out of 262 patients $(18.3 \%)$ in the 2 nd study period $(p<0.001)$. 23 HCW's involved in management of Orthopaedic trauma patients tested positive in the second study period compared to none in the first study period. 17 patients had Hospital-acquired SARS-CoV-2 infection in 2nd study period compared to 7 in 1 st study period. There were more asymptomatic patients for SARS-CoV-2 infection in the 2nd study period.

Conclusion Significant increase in the number of SARS-CoV-2 infection in Orthopaedic trauma patients, and the increased number of HCW's affected with SARS-CoV-2 infection have been the challenges in 2nd study period.
\end{abstract}

Keywords SARS-CoV-2 infection $\cdot$ COVID-19 pandemic $\cdot$ Orthopedics $\cdot$ Trauma

\section{Introduction}

Sameer Aggarwal

drsameer35@yahoo.co.in

Sandeep Patel

sandeepdrpatelortho@gmail.com

Shahnawaz Khan

drskhan2018@gmail.com

Vishal Kumar

drkumarvishal@gmail.com

Siddhartha Sharma

sids82@gmail.com

Mandeep Singh Dhillon

drdhillon@gmail.com

1 Department of Orthopedic Surgery, Postgraduate Institute of Medical Education and Research, Chandigarh, India
The SARS-CoV-2 pandemic has been a problem for optimal patient management across all medical fields. There was a breather after the first wave in India and regular elective surgeries and OPD's had just begun to normalize before the onset of the second wave, which was more of a "tsunami". The second wave struck India badly with a rapid rise in infection rates, hospitalization [1,2]. Although the two waves have hit India in two different years in similar months, there are many differences between them ranging from demography, virus strains, patient management. A significant percentage of the resources and manpower have been diverted to manage SARS-CoV-2-infected patients leading to multiple challenges in management of Orthopaedic trauma patients during the second wave. We aim to present the differences between the first wave and the initial part 
of the second wave in terms of the burden of SARS-CoV-2 infection in patients presenting with orthopaedic trauma and the positivity rate among Health Care Workers (HCWs) involved in the management of Orthopedic trauma patients presenting to the advanced trauma center of a tertiary care center in North India. To the best of our knowledge, no study has been done so far comparing the demographics of Orthopedics patients during the first and second wave of SARSCoV-2 pandemic.

\section{Materials and Methods}

\section{Study Design}

Retrospective cross-sectional study.

\section{Study Period}

We collected data from the trauma registry of our Advanced Trauma Center from April 1, 2021 to the first week of May 2021 (This period corresponds to the peak of the second wave in India). The proportion of rise in cases during this study period was calculated and extrapolated to the first wave to ensure a fair comparison. This period for the first wave corresponded to between June 19, 2020 and October 13, 2020. This fact is graphically represented in Fig. 1. The patients were studied at two different points in time. The time period from June 19, 2020 to October 13, 2020 was labelled "first study period" and the period from April 1, 2021 to the first week of May 2021 was labelled "second study period".

\section{Study Groups}

All the SARS-CoV-2-infected patients with Orthopaedic trauma reporting to our Advanced Trauma Center, Orthopaedic surgeons, residents, the nursing staff and other HCW's involved in the management of Orthopaedic trauma patients at Advanced Trauma Center during the time frames labelled the first and the second were included for evaluation.

\section{Study Setting}

Advanced Trauma Center of our institute.

\section{Outcome Variables}

a. Infection rate among patients in the first and second wave.

b. Infection rate among the Health Care Workers (treating doctors and nursing staff) during the same periods

\section{Active Cases}

\section{(Number of Infected People)}

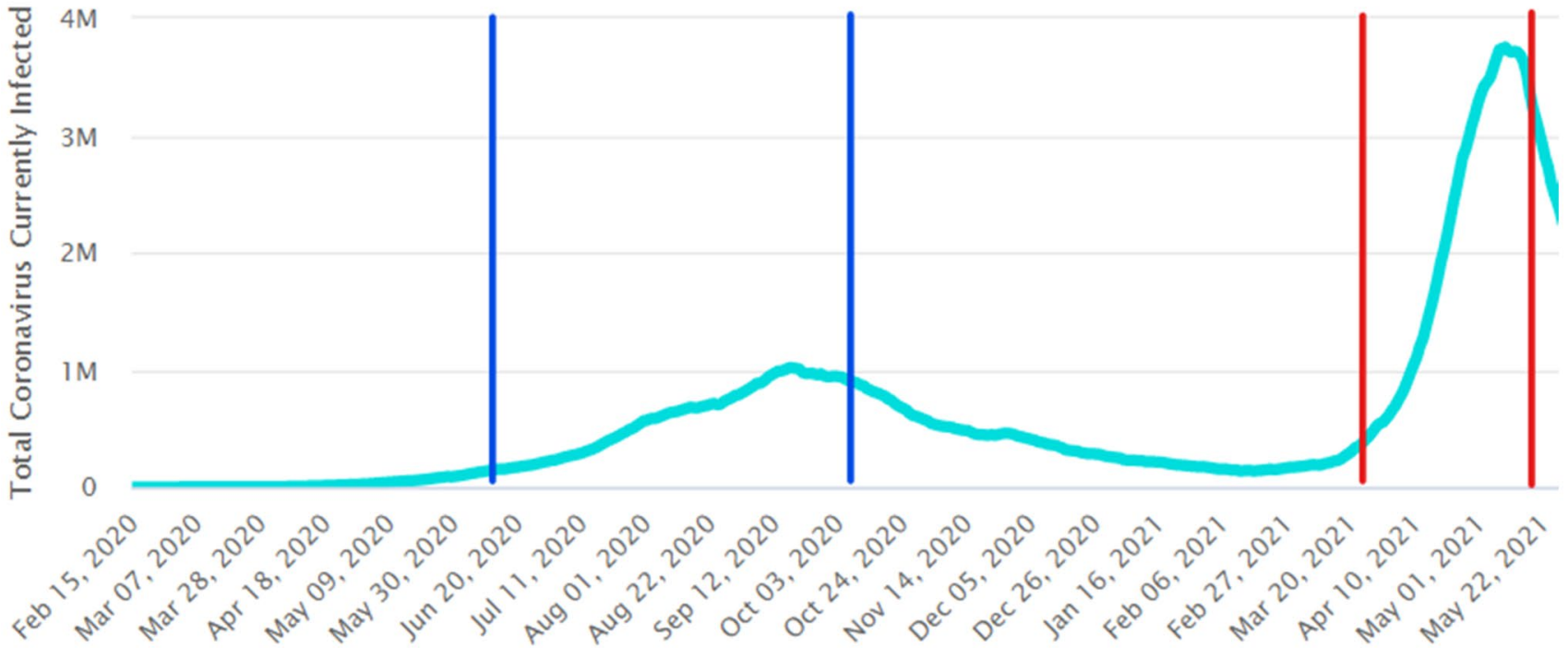

Fig. 1 India's COVID tracker. Reproduced from www.worldometers.info/coronavirus/country/india. The period between blue lines indicated India's first study period and the period between red lines indicates second study period 
c. Incidence of Hospital-acquired SARS-CoV-2 infections in admitted Orthopaedic trauma patients during the two periods studied.

\section{SARS-CoV-2 Testing Protocol}

The patients reporting to our Advanced Trauma Center are screened for symptoms of SARS-CoV-2 at a dedicated screening area. The patients having symptoms such as anosmia, cough, fever, sore throat, diarrhea, respiratory distress, vomiting, history of recent travel and history of contact with a SARS-CoV-2-positive patient are segregated and tested for SARS-CoV-2. Rest of the patients are again differentiated into two groups: the ones requiring emergency surgical management and those in whom surgery can be deferred. The patients who require emergency surgical management undergo GeneXpert for SARS-CoV-2 detection while others undergo mandatory RT-PCR. The positive patients are then shifted to a dedicated COVID wing (a separate building exclusive for management of SARS-CoV-2 infected patients). Only after the test report, the patients are shifted to dedicated wards, HDU, ICU or operation theatre as per the patients' requirement.

Repeat SARS-CoV-2 testing (RT-PCR) is done every 5th day for all admitted patients during their stay in the hospital. This helped us to identify the nosocomial spread of SARS-CoV-2.

\section{Statistics Methods}

All the data were analyzed in SPSS version 21. Chi-square test was used wherever necessary. A $p$ value of less than 0.05 was considered significant.

\section{Results}

We received a total of 852 Orthopaedic trauma patients in the first study duration (the first SARS-CoV-2 peak) and 35 out of these 852 (4.1\%) were positive for SARS-CoV-2 infection. During the second wave, we received a total of 262 patients and 48 patients out of these were positive for SARS-CoV-2 infection (18.3\%). The details of patients are tabulated in Table 1.

\section{Hospital-Acquired SARS-CoV-2 Infection}

Seven patients who were initially SARS-CoV-2 negative on admission were found to be positive on repeating test during their course of hospital stay in the first wave; while 17 patients were found to be positive during their hospital stay in the second wave.
Table 1 Demographics of Orthopaedic trauma patients in the first and second wave of SARS-CoV-2 pandemic

\begin{tabular}{lllc}
\hline & 1 st wave & 2nd wave & $p$ value \\
\hline Total number of Orthopaedic trauma patients & 852 & 262 & \\
Total number of SARS-CoV-2-infected patients & $35(4.1 \%)$ & $48(18.3 \%)$ & $<0.001$ \\
Mean age (years) & $32.6 \pm 5.9$ & $34.8 \pm 4.3$ & 0.05 \\
Sex (M: F) & $28: 7$ & $42: 6$ & 0.353 \\
Mechanism of injury & & & \\
Road traffic accidents & 22 & 34 & 0.444 \\
Fall from height & 8 & 7 & 0.333 \\
Others mechanism of injury & 5 & 7 & 0.970 \\
Injury characteristics & & & 0.187 \\
Open fractures & 26 & 29 & 0.187 \\
Closed fractures & 9 & 19 & \\
Upper limb injury & 9 & 16 & \\
Lower limb injury & 25 & 34 & \\
Spine injury & 4 & 3 & \\
Pelvic injury & 6 & 2 & \\
Associated non-orthopedic injury & 7 & 10 & \\
SARS-CoV-2 infection-related data & & & \\
Patients symptomatic on screening for SARS-CoV-2 symptoms & 23 & 12 & \\
Positive in the first test & 28 & 31 & \\
Hospital-acquired SARS-CoV-2 infection (tested positive subse- & 7 & 17 & \\
quently during hospital stay) & & & \\
\hline
\end{tabular}


Table 2 SARS-CoV-2 Infection among Health Care Workers involved in managing Orthopaedic trauma patients in the first and the second wave of pandemic

\begin{tabular}{llll}
\hline & 1st wave & 2nd wave & $p$-value \\
\hline Total number of treating doctors & 32 & 32 & \\
Total number of nursing staff & 39 & 35 & \\
Number of treating doctors positive & 0 & 16 & $<0.001$ \\
Number of nursing staff positive & 0 & 7 & $<0.013$ \\
\hline
\end{tabular}

Table 3 Vaccination status of the Health Care Workers involved in managing Orthopaedic trauma patients who contracted SARS-CoV-2 Infection

\begin{tabular}{llll}
\hline & $\begin{array}{l}\text { First dose of vac- } \\
\text { cine }\end{array}$ & Fully vaccinated & $\begin{array}{l}\text { Not } \\
\text { vacci- } \\
\text { nated }\end{array}$ \\
\hline $\begin{array}{c}\text { Number of nursing } \\
\text { staff vaccinated } \\
\text { Number of doctors } \\
\text { vaccinated }\end{array}$ & 1 & 0 & 6 \\
\hline
\end{tabular}

\section{Infection Among HCWs}

A total of $71 \mathrm{HCWs}$ (32 doctors and 39 nurses) were involved in management of orthopaedic trauma patients in the first study period while in the second study period, 67 HCWs (32 doctors and 35 nurses) were involved in management of orthopaedic trauma patients (Table 2).

In the first study period, no Health Care Worker involved in the management of Orthopedic trauma patients contacted SARS-CoV-2 infection. However, in the first month of the second wave, 16 doctors and 7 nursing staff involved in the management of Orthopedic trauma patients were diagnosed to be SARS-CoV-2 positive (Table 2). Vaccination status of the HCW's who contracted SARS-CoV-2 is tabulated in Table 3.

\section{Discussion}

Historically, the Spanish flu pandemic has been regarded as the most deadly pandemic and the second wave of the pandemic killed more people than the first wave [3]. Today, we are facing a similar situation all over the world with the second wave of COVID-19 pandemic affecting more persons all over the world [4]. The emergence of new mutants of SARS-CoV-2 which escape the immunity and the abnormal immune response in patients are possible causes for the rapid rise in cases during the second wave $[5,6]$. The situation in India is no different from other countries. The number of cases has gone up significantly with an excessive rise in the hospitalization rates and the death toll [1].

The number of SARS-CoV-2 positive Orthopedic patients has also increased. The number of positive cases in the first month of the second wave is much more than the number of cases during the first wave of pandemic and is statistically significant.

Road traffic accidents remain the major cause of trauma cases even during this pandemic contributing to more than half of the cases in the first and second wave. Most of the patients reported to us had open fractures and required immediate surgical intervention. This could be because the closed injuries were managed conservatively at the peripheral health care centers. Only the complex trauma and the open fractures which required a multidisciplinary approach were referred to us.

During the first wave, 23 out of 35 SARS-CoV-2 patients with trauma were symptomatic on SARS-CoV-2 screening $(65.7 \%)$ which made suspicion of diagnosis much easier. The most striking feature of this second wave was a rise in the number of asymptomatic patients. Only $25 \%$ of our patients had symptoms of SARS-CoV-2. Similar findings have been reported worldwide in other studies [7-9]. A high index of suspicion is, therefore, necessary to manage this second wave. Many patients may become asymptomatic carriers of SARS-CoV-2.

In our study in both waves of the pandemic, we found out that many patients were diagnosed to be SARS-CoV-2 positive during their treatment at the hospital after an initial negative result during admission. This highlights the importance of repeated testing of patients at regular intervals. The possible three reasons for this may be: (1) a single negative RT-PCR report does not rule out SARS-CoV-2 infection due to the low sensitivity of RT-PCR test, (2) longer incubation period, and (3) patient may have acquired from family members and attendants who accompany trauma patients. Across many hospitals in India which have a huge patient load, patients' family members and attendants are usually present with the patient in the wards who provide help to manage basic hygiene and feeding along with the depleted number of support HCW's available. The family members and attendants who roam in the hospital campus, canteens could have acquired the infection. Additional testing of patient attenders is a solution, but will add to the already overburdened numbers of existing testing capacity.

Another important challenge faced during this second wave is the positivity rate among health care providers. As many as 23 HCW's (16 doctors and 7 nursing staff) involved in the management of Orthopedic trauma patients have been diagnosed to have SARS-CoV-2 opposed to none in the first wave. This highlights the fact that the transmission rate is extremely high in the second wave. Nosocomial transmission of SARS-CoV-2 
remains a big challenge today [10]. This infection among the HCW's could be community-acquired or nosocomial. However, due to the massive number of cases during this period, contact tracing of the source is difficult and has not been successful. Despite the vaccination program being implemented in January for health care workers, there has been hesitancy in taking the vaccination. It is evident that amongst all the HCW's infected with SARS-CoV-2, only one nursing staff and four doctors had received one shot of vaccine prior to infection and only one doctor was fully vaccinated ( 2 doses 1 month apart) of vaccine. Rana et al. [11] have recently published the data of HCW's infected at our tertiary center and have noted that breakthrough infections was significantly less among the HCWs who received complete dose of vaccination ( 2 doses 1 month apart). The increased positivity rate among the HCW's could be due to endemicity of disease or evolution of new strains which evade the immune system and the memory cells generated by the vaccines, a fact which needs further investigation [12]. With time, there has been a behavioral fatigue among people and HCW's and they have not been following COVID appropriate behaviour. This behavioral fatigue could also be responsible for an increase in the infection rate among the HCWs [13]. Various studies in the past have emphasized the fact that noncompliance with hygiene practices of appropriate standards have resulted in high infectivity and mortality among HCWs and their family members [14-16]. Therefore, repeated screening of health care workers is the need of the hour [17] and some centers in the west are advising weekly mandatory COVID testing for the HCW's. Newer tests which involve the use of saliva for detection of SARS-CoV-2 can be used by healthcare professionals as a mode of self-testing [18]. This is a convenient method and does not involve exposure to other people. The health care professional can test themselves every week, and if found positive can self-isolate themselves. The hesitancy for vaccination is improving with time, and after the second wave, we have seen almost all health care workers volunteering for getting vaccinated.

The rise in SARS-CoV-2 infections is expected in other departments too. This exponential rise in the number of SARS-CoV-2 cases during this second wave has prompted the hospital administration to expand the COVID care facilities. In addition to dedicated COVID hospitals, normal wards are being converted into facilities for the management of SARS-CoV-2 patients. Due to these circumstances, the management of non-COVID patients has taken a hit. There is a need to be more vigilant in screening and isolating the SARS-CoV-2-infected patients from normal patients in emergency areas to prevent the ER from becoming hotspots of SARS-CoV-2 cases.

\section{Limitations of the Study}

However, this study is not without limitations. Foremost is the fact that the second wave is still continuing. The patient attendants were not tested for SARS CoV2 infection making it difficult to assess the exact source of infection. This study did not involve contact tracing of the patients. We took only Orthopaedic trauma patients into consideration. However, there were patients of other departments at the trauma center who could have been a potential source of infection. We included only the doctors and the nursing staff in this study. However, the sanitary staffs and the hospital attendants were not included in this study.

\section{Future Directions}

This study can be replicated in a large study population involving other nearby tertiary care centers with a longer duration. The nosocomial spread of SARS-CoV-2 infection is an important aspect that needs to be studied in future studies and more robust measures need to be taken to control the same in the developing countries. With the number of cases coming down, we cannot afford to be complacent as the new mutants of SARS COV-2 virus have been reported. Vaccination of all HCWs and use of personal protection and sanitary methods will help us overcome the subsequent waves.

\section{Conclusion}

The challenges in the management of Orthopedic trauma patients in the second wave of the pandemic have been the significant increase in the number of SARS-CoV-2 infection, asymptomatic SARS-CoV-2 patients, nosocomial acquired SARS-CoV-2 infection and the increased number of HCW's affected with SARS-CoV-2 infection. Dedicated isolation areas in ER, need for repeated testing, testing of patients' attendants, weekly testing of HCW's are possible solutions.

Funding This research did not receive any specific grant from funding agencies in public, commercial, or not-for profit sectors.

\section{Declarations}

Conflict of interest The authors have no conflict of interest to declare.

Ethical standard statement This article does not contain any studies with human or animal subjects performed by the any of the authors.

Informed consent For this type of study informed consent is not required. 


\section{References}

1. India: WHO Coronavirus Disease (COVID-19) dashboard with vaccination data. (2021). https://covid19.who.int. Accessed 28 May 2021.

2. Kumar, S. (2021). Second wave of COVID-19: Emergency situation in India. Journal of Travel Medicine. https://doi.org/10.1093/ $\mathrm{jtm} / \mathrm{taab} 082$

3. Staub, K., Jüni, P., Urner, M., et al. (2021). Public health interventions, epidemic growth, and regional variation of the 1918 influenza pandemic outbreak in a Swiss Canton and its greater regions. Annals of Internal Medicine, 174(4), 533-539. https:// doi.org/10.7326/M20-6231

4. Iftimie, S., López-Azcona, A. F., Vallverdú, I., et al. (2021). First and second waves of coronavirus disease-19: A comparative study in hospitalized patients in Reus, Spain. PLOS ONE, 16, 3. https:// doi.org/10.1371/journal.pone.0248029

5. Hussein, O. (2020). Second wave of Covid-19 is determined by immune mechanism. Medical Hypotheses, 144, 110238. https:// doi.org/10.1016/j.mehy.2020.110238

6. Ko, K., Nagashima, S., Bunthen, E., et al. (2021). Molecular characterization and the mutation pattern of SARS-CoV-2 during first and second wave outbreaks in Hiroshima, Japan. PLOS ONE, 16(2), e0246383. https://doi.org/10.1371/journal.pone.0246383

7. Wu, Z. Y. (2020). Asymptomatic and pre-symptomatic cases of COVID-19 contribution to spreading the epidemic and need for targeted control strategies. Zhonghua Liu Xing Bing Xие Za Zhi Zhonghua Liuxingbingxue Zazhi., 41(00), E036. https://doi.org/ 10.3760/cma.j.cn112338-20200406-00517

8. Lampl, B. M. J., \& Salzberger, B. (2020). Changing epidemiology of COVID-19. GMS Hygiene and Infection Control, 15, Doc27. https://doi.org/10.3205/dgkh000362

9. Jain, V. K., Iyengar, K. P., \& Vaishya, R. (2021). Differences between First wave and Second wave of COVID-19 in India. Diabetes \& Metabolic Syndrome Published Online. https://doi. org/10.1016/j.dsx.2021.05.009

10. Abbas, M., Robalo, N. T., Martischang, R., et al. (2021). Nosocomial transmission and outbreaks of coronavirus disease 2019: The need to protect both patients and healthcare workers.
Antimicrobial Resistance and Infection Control. https://doi.org/ 10.1186/s13756-020-00875-7

11. Hacisuleyman, E., Hale, C., et al. (2021). Vaccine breakthrough infections with SARS-CoV-2 variants. The New England Journal of Medicine. https://doi.org/10.1056/NEJMc2107808

12. Vaidyanathan, G. (2021). Coronavirus variants are spreading in India - what scientists know so far. Nature, 593(7859), 321-322. https://doi.org/10.1038/d41586-021-01274-7

13. Harvey, N. (2020). Behavioral fatigue: Real phenomenon, naïve construct, or policy contrivance? Frontiers in Psychology, 2020, 11. https://doi.org/10.3389/fpsyg.2020.589892

14. Iyengar, K. P., Ish, P., Upadhyaya, G. K., Malhotra, N., Vaishya, R., \& Jain, V. K. (2020). COVID-19 and mortality in doctors. Diabetes and Metabolic Syndrome: Clinical Research and Reviews, 14(6), 1743-1746. https://doi.org/10.1016/j.dsx.2020.09.003

15. Alajmi, J., Jeremijenko, A. M., Abraham, J. C., et al. (2020). COVID-19 infection among healthcare workers in a national healthcare system: The Qatar experience. International Journal of Infectious Diseases, 100, 386-389. https://doi.org/10.1016/j. ijid.2020.09.027

16. Bandyopadhyay, S., Baticulon, R. E., Kadhum, M., et al. (2020). Infection and mortality of healthcare workers worldwide from COVID-19: A systematic review. BMJ Global Health, 5(12), e003097. https://doi.org/10.1136/bmjgh-2020-003097

17. Rivett, L., Sridhar, S., Sparkes, D., et al. (2020). Screening of healthcare workers for SARS-CoV-2 highlights the role of asymptomatic carriage in COVID-19 transmission. eLife, 2020, 9. https://doi.org/10.7554/eLife.58728

18. Hung, K.-F., Sun, Y.-C., Chen, B.-H., et al. (2020). New COVID19 saliva-based test: How good is it compared with the current nasopharyngeal or throat swab test? Journal of the Chinese Medical Association JCMA, 83(10), 891-894. https://doi.org/10.1097/ JCMA.0000000000000396

Publisher's Note Springer Nature remains neutral with regard to jurisdictional claims in published maps and institutional affiliations. 